This is the author's final, peer-reviewed manuscript as accepted for publication. The publisher-formatted version may be available through the publisher's web site or your institution's library.

\title{
Competing hydrogen-bond and halogen-bond donors in crystal engineering
}

Christer B. Aakeröy, Sheelu Panikkattu, Prashant D. Chopade, John Desper

\section{How to cite this manuscript}

If you make reference to this version of the manuscript, use the following information:

Aakeröy, C. B., Panikkattu, S., Chopade, P. D., \& Desper, J. (2013). Competing hydrogen-bond and halogen-bond donors in crystal engineering. Retrieved from http://krex.ksu.edu

\section{Published Version Information}

Citation: Aakeröy, C. B., Panikkattu, S., Chopade, P. D., \& Desper, J. (2013). Competing hydrogen-bond and halogen-bond donors in crystal engineering. CrystEngComm, 15(16), 3125-3136.

Copyright: @ The Royal Society of Chemistry 2013

Digital Object Identifier (DOI): doi:10.1039/c2ce26747k

Publisher's Link: http://pubs.rsc.org/en/Content/ArticleLanding/2013/CE/c2ce26747k

This item was retrieved from the K-State Research Exchange (K-REx), the institutional repository of Kansas State University. K-REx is available at http://krex.ksu.edu 


\title{
Competing hydrogen-bond and halogen-bond donors in crystal engineering
}

\author{
Christer B. Aakeröy, ${ }^{a}$ Sheelu Panikkattu, ${ }^{a}$ Prashant D. Chopade, ${ }^{a}$ and John Desper ${ }^{a}$
}

5 In order to study the structure-directing competition between hydrogen- and halogen-bond donors we have synthesized two ligands, 3,3'-azobipyridine and 4,4'-azobipyridine, and co-crystallized them with a series of bi-functional donor molecules comprising an activated halogen-bond donor (I or Br) as well as a hydrogen-bond donor (acid, phenol or oxime) on the same backbone. Based on the subsequent singlecrystal analysis, 5 of 6 co-crystals of 3,3'-azobipyridine are assembled using hydrogen bonds as the 10 primary driving force accompanied by weaker secondary $(\mathrm{C}-\mathrm{X} \cdots \mathrm{O})$ interactions. However, in 5 out of the 6 co-crystals of 4,4 '-azobipyridine, both hydrogen bonds $(\mathrm{O}-\mathrm{H} \cdots \mathrm{N})$ and halogen bonds $(\mathrm{C}-\mathrm{X} \cdots \mathrm{N})$ are present as comparable structure-directing interactions leading to 1-D chains. Since the charges on the acceptor sites in 3,3'-and 4,4'-azobipyridine are very similar, i.e. -174 and $-172 \mathrm{~kJ} / \mathrm{mol}$ respectively, the observed difference in binding behaviour highlights the importance of binding-site location on the 15 acceptor molecules (anti-parallel in 3,3'-azobipyridine and co-linear in 4,4'-azobipyridine) as a direct influence over the structural balance between hydrogen- and halogen-bond donors.

\section{Introduction}

Many hydrogen-bond based synthons ${ }^{1}$ such as acid...pyridine ${ }^{2}$, acid $\cdots$ amide $^{3}$, phenol $\cdots$ pyridine ${ }^{4}$, oxime $\cdots \mathrm{N}(\text { heterocycle })^{5}$ have 20 been explored extensively as robust and reliable tools for crystal engineering and supramolecular synthesis. Recently, halogen bonds ${ }^{6}$ which can play important roles in areas such as biochemistry ${ }^{7}$, medicinal chemistry ${ }^{8}$, and material science, ${ }^{9}$ have found uses in crystal engineering because these 25 interactions have properties that parallel those of hydrogen bonds in terms of directionality and strength. ${ }^{10,11}$ Typical hydrogen-bond strength ranges from approximately 4-60 $\mathrm{kJ} / \mathrm{mol}^{12}$ while halogen bonds range from $5-180 \mathrm{~kJ} / \mathrm{mol}$ (the strong interaction $\mathrm{I}_{2} \cdots \mathrm{I}^{-}$in $\mathrm{I}_{3}{ }^{-}$is the extreme) ${ }^{13}$ Consequently, 30 iodine/bromine suitably 'activated' through a fluorinated backbone should be capable of competing with hydrogen bonds in a supramolecular reaction. ${ }^{14}$

The combination of hydrogen bonding (HB) and halogen bonding (XB) is gaining a lot of importance in crystal 35 engineering, ${ }^{15}$ and in order to investigate the structuredirecting balance between $\mathrm{HBs}$ and $\mathrm{XBs}$ we decided to employ a set of bi-functional donor molecules equipped with one $\mathrm{HB}$ donor and one $\mathrm{XB}$ donor attached to the same molecular backbone; 4-iodotetrafluorobenzoic acid $(\mathrm{COOH}-$

$40 \mathrm{I}$, 4-bromotetrafluorobenzoic acid (COOH-Br), 4iodotetrafluorophenol (OH-I), 4-bromotetrafluorophenol $(\mathrm{OH}-$ $\mathrm{Br})$ 4-iodotetrafluoroaldoxime (Ox-I) and 4bromotetrafluoroaldoxime (Ox-Br) (Scheme 1). These probe molecules were subsequently co-crystallized with two 45 isomeric symmetric acceptors 3,3'-and 4,4'-azobipyridine (3,3'-azpy and 4,4'-azpy, respectively) (Scheme 1).

\section{Donors}<smiles>O=C(O)c1c(F)c(F)c(C(=O)O)c(F)c1F</smiles><smiles>O=C(O)c1c(F)c(F)c(Br)c(F)c1F</smiles><smiles>Oc1c(F)c(F)c(Br)c(Br)c1F</smiles><smiles>O/N=C/c1c(F)c(F)c(Cl)c(F)c1F</smiles><smiles></smiles><smiles></smiles>

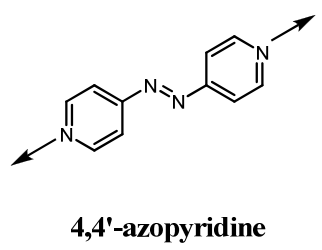

Scheme 1. Bi-functional donors and isomeric symmetric acceptors 50 In this study, we investigate the following; 
1) Competition between $\mathrm{HB}$ and $\mathrm{XB}$ donors for the pyridine nitrogen atoms which are capable of forming both hydrogen bonds and halogen bonds, (Scheme 2).

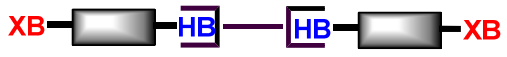

(A) $\mathrm{HB}>\mathrm{XB}$

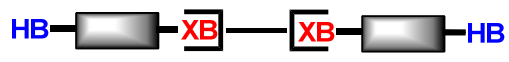

(B) $\mathrm{HB}<\mathrm{XB}$

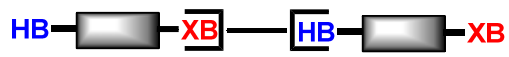

(C) $\mathrm{HB} \sim \mathrm{XB}$

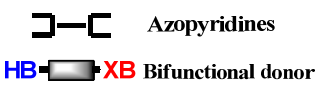

Scheme 2. Postulated outcomes in our study

2) Possible influence of geometric differences of the donors and acceptors on the competition and overall structural outcome.

3) Participation of auxiliary potential acceptors like carbonyl oxygen atoms, hydroxyl oxygen atoms and azo nitrogen atoms (Scheme 3) in weaker interactions with halogen atoms.<smiles></smiles><smiles>[R]O[X]([2H])c1ccccc1</smiles>

15

Scheme 3. Potential secondary interactions between halogen and oxygen/azo-nitrogen

In Scheme 2, we have outlined the postulated structural outcomes of the co-crystallizations as a function of the 20 relative strength and supramolecular efficiency of HB vs. XB in this series of co-crystallizations.

It is well known that electrostatic charge is important for predicting or rationalizing molecular recognition events, ${ }^{16}$ but in this study, the two acceptor molecules have rather similar
25 electrostatic potentials of interaction as indicated by the charges 174 and $172 \mathrm{~kJ} / \mathrm{mol}$ for 3,3'-azpy and 4,4'-azpy respectively, for which the maxima and minima in the molecular electrostatic potential surface $(0.002$ e au-1) were determined using a positive charge in vacuum as the probe 30 after optimizing their molecular geometries using DFT (B3LYP, 6-31++G**) basis set. There is therefore no real charge-based 'bias' in the two acceptors. However, there is a geometric difference since the binding sites differ in their relative orientation, i.e. in case of 3,3'-azpy, the pyridine

35 nitrogen atoms are aligned anti-parallel whereas in 4,4'-azpy they are co-linear with respect to each other (Scheme 1). It is conceivable that this difference, may affect the primary molecular recognition events, which can subsequently lead to the formation of distinctly different supramolecular 40 assemblies.

\section{Experimental set up}

A library of six bifunctional halogen-hydrogen bond donors and two symmetric acceptors (Scheme 1) was employed. 3,3'-

45 azobipyridine and 4,4'-azobipyridine, ${ }^{17}$ 4iodotetrafluorobenzoic acid and 4-bromotetrafluorobenzoic acid $^{18}$ were synthesised as reported. 4-iodotetrafluorophenol, 4-iodotetrafluoroaldoxime, 4-bromotetrafluoroaldoxime were synthesised in one-step from their corresponding 450 halotetrafluoroaldehydes via greener solvent-assisted grinding method developed in our group. Synthesis of all the HB-XB donors is included in the supporting information. 4bromotetrafluorophenol was purchased from Sigma Aldrich. The donors and acceptors were combined in stoichiometric 55 amounts giving a total of 12 co-crystal combinations, the synthesis of which is reported in Table 1 . Single crystals were obtained upon slow evaporation of the solvent, and we were able to obtain crystals suitable for single-crystal diffraction for all twelve reactions. The structural outcomes are summarized 60 below. 
Cite this: DOI: 10.1039/Coxxooooox

www.rsc.org/xxxxxx

PAPER

Table 1. Synthesis of 3,3'-and 4,4'-azobipyridine co-crystals

\begin{tabular}{|c|c|c|c|c|c|}
\hline Acceptors & Donors & Co-crystal abbreviation & Mole ratio & Solvent/ method & Melting points ${ }^{\circ} \mathrm{C}$ \\
\hline \multirow{6}{*}{ 3,3'-azobipyridine } & 4-iodotetrafluorobenzoicacid & 3,3'-azpy:COOH-I & $1: 1$ & $\begin{array}{l}\text { Ethanol/slow } \\
\text { evaporation }\end{array}$ & $182-185$ \\
\hline & 4-bromotetrafluorobenzoicacid & 3,3'-azpy:COOH-Br & $1: 1$ & $\begin{array}{c}\text { Ethanol/ slow } \\
\text { evaporation }\end{array}$ & $155-157$ \\
\hline & 4-iodotetrafluorophenol & 3,3'-azpy:OH-I & $1: 1$ & $\begin{array}{l}\text { Ethanol/slow } \\
\text { evaporation }\end{array}$ & $125-127$ \\
\hline & 4-bromotetrafluorophenol & 3,3'-azpy:OH-Br & $1: 1$ & $\begin{array}{l}\text { Ethanol/slow } \\
\text { evaporation }\end{array}$ & $170-173$ \\
\hline & 4-iodotetrafluoroaldoxime & 3,3'-azpy:Ox-I & $1: 1$ & $\begin{array}{l}\text { Ethanol/slow } \\
\text { evaporation }\end{array}$ & $145-148$ \\
\hline & 4-bromotetrafluoroaldoxime & 3,3'-azpy:Ox-Br & $1: 1$ & $\begin{array}{c}\text { Ethanol/ slow } \\
\text { evaporation }\end{array}$ & $105-108$ \\
\hline \multirow{6}{*}{ 4,4'-azobipyridine } & 4-iodotetrafluorobenzoicacid & 4,4'-azpy:COOH-I & $1: 1$ & $\begin{array}{l}\text { Ethanol/slow } \\
\text { evaporation }\end{array}$ & $220-222$ \\
\hline & 4-bromotetrafluorobenzoicacid & 4,4'-azpy:СОOH-Br & $1: 1$ & $\begin{array}{l}\text { Ethanol/slow } \\
\text { evaporation }\end{array}$ & $190-191$ \\
\hline & 4-iodotetrafluorophenol & 4,4'-azpy:OH-I & $1: 1$ & $\begin{array}{l}\text { Ethanol/slow } \\
\text { evaporation }\end{array}$ & $163-165$ \\
\hline & 4-bromotetrafluorophenol & 4,4'-azpy:OH-Br & $1: 1$ & $\begin{array}{l}\text { Ethanol/slow } \\
\text { evaporation }\end{array}$ & $133-136$ \\
\hline & 4-iodotetrafluoroaldoxime & 4,4'-azpy:Ox-I & $1: 1$ & $\begin{array}{l}\text { Ethanol/slow } \\
\text { evaporation }\end{array}$ & $115-116$ \\
\hline & 4-bromotetrafluoroaldoxime & 4,4'-azpy:Ox-Br & $1: 1$ & $\begin{array}{l}\text { Ethanol/slow } \\
\text { evaporation }\end{array}$ & $125-126$ \\
\hline
\end{tabular}

\section{X-Ray Crystallography}

Datasets were collected on a Bruker SMART APEX II system 5 with Mo radiation (3,3'-azpy:COOH-I, 3,3'-azpy:OH-I ) 3,3'-azpy:OH-Br，3,3'-azpy:Ox-I，3,3'-azpy:Ox-Br，4,4'azpy:COOH-I, 4,4;azpy:OH-Br, 4,4'azpy:Ox-I or a Bruker Kappa APEX II system with $\mathrm{Cu}$ radiation (3,3'-azpy:COOH$\mathrm{Br}$, 4,4'-azpy:COOH-Br, 4,4'-azpy:OH-I, 4,4'-azpy:Ox$10 \mathrm{Br})$ at $120 \mathrm{~K}$ using APEX2 software. ${ }^{19}$ An Oxford Cryostream 700 low-temperature device was used to control temperature. $\mathrm{MoK} \alpha$ radiation was used. Initial cell constants were found by small widely separated "matrix" runs. Data collection strategies were determined using COSMO. ${ }^{20}$ Scan speeds and 15 scan widths were chosen based on scattering power and peak rocking curves.

Unit-cell constants and orientation matrices were improved by least-squares refinement of reflections threshold from the entire dataset. Integrations were performed with SAINT, ${ }^{21}$

20 using these improved unit cells as a starting point. Precise unit cell constants were calculated in SAINT from the final merged datasets. Lorenz and polarization corrections were applied. Absorption corrections was applied using SADABS ${ }^{22}$ Datasets were reduced with SHELXTL. ${ }^{23}$ The structures were 25 solved by direct methods without incident. Coordinates for all oxime and phenol hydrogen atoms were allowed to refine. All other hydrogen atoms were assigned to idealized positions and were allowed to ride. Isotropic thermal parameters for the hydrogen atoms were constrained to be $1.5 \mathrm{x}$ (methyl) / $1.2 \mathrm{x}$ 30 (all other) that of the connected atom.

3,3'-azpy:COOH-I Two orientations for the haloacid were located, in roughly head-to-tail positions. Geometry for the two components was restrained to similarity by using the SHELXL "SAME" command. Thermal parameters of closely 35 located atoms were pairwise constrained using the SHELXL "EADP" command. Coordinates of the ammonium proton H11 were allowed to refine.

3,3'-azpy:COOH-Br Coordinates for the carboxylic acid proton $\mathrm{H} 21$ were allowed to refine.

40 3,3'-azpy:OH-I Coordinates for the carboxylic acid proton $\mathrm{H} 21$ were allowed to refine.

3,3'-azpy:OH-Br Coordinates for the phenol protons H31 and H41 were allowed to refine..

3,3'-azpy:Ox-I Coordinates for the oxime proton $\mathrm{H} 27$ 45 were allowed to refine.

3,3'-azpy:Ox-Br Coordinates for the oxime proton $\mathrm{H} 17$ were allowed to refine

4,4'-azpy:COOH-I The sample was a racemic twin, and populations of the two components was parameterized using 50 the SHELXL "TWIN" and "BASF" commands. Two orientations for the haloacid were located, in roughly head-totail positions. Geometry for the two components was restrained to similarity by using the SHELXL "SAME" command. Thermal parameters of closely located atoms were ${ }_{55}$ pairwise constrained using the SHELXL "EADP" command. 
Thermal parameters for the nearly inversion-related pyridines N11-C16 and N21-C26 were also constrained using the "EADP" command, and these thermal parameters were restrained to approximately the same values using the 5 SHELXL "SIMU" command. The ammonium proton H11 was placed in an idealized geometry and allowed to ride on its parent nitrogen atom.

4,4'-azpy: $\mathrm{COOH}-\mathrm{Br}$ The asymmetric unit contains two acid / base pairs, each of which was assigned to a SHELXL RESI 10 due for consistent labelling purposes. Two orientations for each halo acid were located, in roughly head-to-tail positions. Similarly, two closely related orientations for the amine were found. Geometry for all four acid components was restrained to similarity by using the SHELXL "SAME" command.

${ }_{15}$ Geometry for all four base components was also restrained to similarity by using the SHELXL "SAME" command. Thermal parameters of closely located atoms were pairwise constrained using the SHELXL "EADP" command.

4,4'-azpy:OH-I The phenol proton H31 was placed in an 20 idealized geometry and allowed to ride on its parent oxygen atom.

4,4'-azpy:OH-Br Coordinates for the phenol proton H11 were allowed to refine.

4,4'-azpy:Ox-I Coordinates for the oxime proton H37 were 25 allowed to refine.

4,4'-azpy:Ox-Br Coordinates for the oxime proton $\mathrm{H} 27$ were allowed to refine.

Selected hydrogen and halogen-bond geometries are shown 30 in Table 2 and 3 respectively. The crystallographic parameters of 3,3'- and 4,4'-azobipyridine co-crystals are listed in Table $4 \mathrm{a}$ and $4 \mathrm{~b}$, respectively.

35 Table 2 Hydrogen-bond geometries in co-crystals of 3,3'- and 4,4'-azobipyridine ${ }^{\mathrm{a}}$

\begin{tabular}{|c|c|c|c|c|c|}
\hline Structure & $\mathrm{D}-\mathrm{H} \ldots \mathrm{A} / \AA$ & $\mathrm{D}-\mathrm{H} / \AA$ & $\mathrm{H} \cdots \mathrm{A} / \AA$ & $\mathrm{D} \cdots \mathrm{A} / \AA$ & $\mathrm{D}-\mathrm{H} \cdots \mathrm{N} /{ }^{\circ}$ \\
\hline 3,3'-azpy:COOH-I & $\mathrm{N}(11)-\mathrm{H}(11) \cdots \mathrm{O}(21 \mathrm{~A}) \_\# 1$ & $1.22(2)$ & $1.32(2)$ & $2.536(2)$ & $174(2)$ \\
\hline 3,3'-azpy:COOH-Br & $\mathrm{O}(21)-\mathrm{H}(21) \cdots \mathrm{N}(11) \_\# 1$ & $1.14(3)$ & $1.41(3)$ & $2.549(2)$ & $177(3)$ \\
\hline 3,3'-azpy:OH-I & $\mathrm{O}(21)-\mathrm{H}(21) \cdots \mathrm{N}(11) \# 1$ & $0.90(2)$ & $1.75(2)$ & $2.6162(15)$ & $159(2)$ \\
\hline \multirow[t]{2}{*}{ 3,3'-azpy:OH-Br } & $\mathrm{O}(31)-\mathrm{H}(31) \cdots \mathrm{N}(11)$ & $0.88(2)$ & $1.77(2)$ & $2.6224(16)$ & $163.2(18)$ \\
\hline & $\mathrm{O}(41)-\mathrm{H}(41) \cdots \mathrm{N}(21)$ & $0.88(2)$ & $1.73(2)$ & $2.5963(15)$ & $166.8(18)$ \\
\hline 3,3'-azpy:Ox-I & $\mathrm{O}(27)-\mathrm{H}(27) \cdots \mathrm{N}(27) \_\# 1 \_\# 2$ & $0.72(2)$ & $2.18(2)$ & $2.8460(17)$ & $153(2)$ \\
\hline 3,3'-azpy:Ox-Br & $\mathrm{O}(17)-\mathrm{H}(17) \cdots \mathrm{N}(21)$ & $0.76(4)$ & $1.95(4)$ & $2.708(3)$ & $178(4)$ \\
\hline \multirow[t]{2}{*}{ 4,4'-azpy:COOH-I } & $\mathrm{N}(11)-\mathrm{H}(11) \cdots \mathrm{O}(31 \mathrm{~B}) \# 1$ & 0.88 & 1.75 & $2.605(9)$ & 164.3 \\
\hline & & 0.88 & 1.73 & $2.598(6)$ & 167.5 \\
\hline \multirow[t]{4}{*}{ 4,4'-azpy:COOH-Br } & 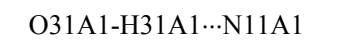 & 0.84 & 1.76 & $2.566(10)$ & 158.8 \\
\hline & O31B1-H31B1N21B1 \#1 & 0.84 & 1.84 & $2.67(3)$ & 167.6 \\
\hline & $\mathrm{O} 31 \mathrm{~A} 2-\mathrm{H} 31 \mathrm{~A} 2 \cdots \mathrm{N} 11 \mathrm{~A} 2$ & 0.84 & 1.75 & $2.587(9)$ & 170.0 \\
\hline & O31B2-H31B2 $\cdots$ N21B2_\#2 & 0.84 & 1.83 & $2.67(4)$ & 175.3 \\
\hline 4,4'-azpy:OH-I & $\mathrm{O}(31)-\mathrm{H}(31) \cdots \mathrm{N}(11)$ & 0.84 & 1.84 & $2.614(4)$ & 152.3 \\
\hline 4,4'-azpy:OH-Br & $\mathrm{O}(31)-\mathrm{H}(31) \cdots \mathrm{N}(11)$ & $0.83(2)$ & $1.86(2)$ & $2.6342(16)$ & $155(2)$ \\
\hline 4,4'-azpy:Ox-I & $\mathrm{O}(37)-\mathrm{H}(37) \cdots \mathrm{N}(11)$ & $0.82(2)$ & $1.90(2)$ & $2.7052(19)$ & $169(2)$ \\
\hline 4,4'-azpy:Ox-Br & $\mathrm{O}(27)-\mathrm{H}(27) \cdots \mathrm{N}(11) \_\# 1$ & $0.94(6)$ & $1.79(6)$ & $2.704(6)$ & $162(6)$ \\
\hline
\end{tabular}

${ }^{a}$ Symmetry codes: 3,3'-azpy:COOH-I \#1 -x-1,-y,-z+2. 3,3'-azpy:COOH-Br \#1 -x+2,-y+1,-z. 3,3'-azpy:OH-I \#1 -x+1,-y+1,-z+1. 3,3'-azpy:Ox-I \#1 x,-y,-z+2 \#2 -x+3,-y+1,-z. 3,3'-azpy:Ox-Br \#1 -x,-y,-z-1. 4,4'-azpy:COOH-I \#1 x+2,y-2,z+1. 4,4'-azpy:COOH-Br \#1 x-1,y+1,z+1 \#2 x+2,y+1,z+1 4,4'-azpy:Ox-Br \#1-x+2,-y+3,-z+1 
Table 3 Halogen-bond geometries in co-crystals of 3,3'-and 4,4' azobipyridine ${ }^{\text {a }}$

\begin{tabular}{|c|c|c|c|}
\hline Structure & $\mathrm{C}-\mathrm{X} \cdots \mathrm{A}$ & $\mathrm{X} \cdots \mathrm{A} / \AA$ & $\mathrm{C}-\mathrm{X} \cdots \mathrm{A} /{ }^{\circ}$ \\
\hline 3,3'-azpy:COOH-I & $\mathrm{C}-(24 \mathrm{~A})-\mathrm{I}(1) \cdots \mathrm{O}(22 \mathrm{~A}) \_\# 2$ & $2.8694(13)$ & $170.66(6)$ \\
\hline 3,3'-azpy:COOH-Br & $\mathrm{C}-(24)-\mathrm{Br}(1) \cdots \mathrm{O}(22) \_\# 2$ & $2.8792(17)$ & $172.23(7)$ \\
\hline 3,3'-azpy:OH-I & $\mathrm{C}(24)-\mathrm{I}(1) \cdots \mathrm{O}(21) \_\# 2$ & $3.0913(10)$ & $156.81(4)$ \\
\hline \multirow[t]{2}{*}{ 3,3'-azpy:OH-Br } & $\mathrm{C}(34)-\mathrm{Br}(1) \cdots \mathrm{O}(31) \ldots \# 1$ & $3.1138(11)$ & $156.32(5)$ \\
\hline & $\mathrm{C}(44)-\mathrm{Br}(2) \cdots \mathrm{O}(41) \_\# 2$ & $3.0750(10)$ & $158.68(5)$ \\
\hline 3,3'-azpy:Ox-I & $C(24)-I(1) \cdots N(11)$ & $2.8279(12)$ & $174.75(4)$ \\
\hline 3,3'-azpy:Ox-Br & $C(14)-B r(1) \cdots O(17) \_\# 1$ & $3.0557(19)$ & $158.49(9)$ \\
\hline 4,4'-azpy:COOH-I & $\mathrm{C}(34 \mathrm{~A})-\mathrm{I}(1 \mathrm{~A}) \cdots \mathrm{N}(21)$ & $2.796(5)$ & $173.4(3)$ \\
\hline 4,4'-azpy:COOH-Br & $\mathrm{C}(34 \mathrm{~A} 1)-\mathrm{Br}(1 \mathrm{~A} 1) \cdots \mathrm{N}(21 \mathrm{~A} 1) \_\# 1$ & $2.802(7)$ & $176.9(4)$ \\
\hline 4,4'-azpy:OH-I & $\mathrm{C}(34)-\mathrm{I}(1) \cdots \mathrm{N}(21) \# 1$ & $2.960(3)$ & $165.98(11)$ \\
\hline 4,4'-azpy:OH-Br & $\mathrm{C}(34)-\mathrm{Br}(1) \cdots \mathrm{N}(21) \_\# 1$ & $2.9717(12)$ & $165.95(5)$ \\
\hline 4,4'-azpy:Ox-I & $\mathrm{C}(34)-\mathrm{I}(1) \cdots \mathrm{N}(21) \_\# 1$ & $2.8200(15)$ & $178.38(5)$ \\
\hline 4,4'-azpy:Ox-Br & $\mathrm{C}(24)-\mathrm{Br}(1) \cdots \mathrm{N}(14) \_\# 1$ & $3.395(4)$ & $171.94(17)$ \\
\hline
\end{tabular}

${ }^{a}$ Symmetry codes 3,3'-azpy:COOH-I \#2 x+1,-y+1/2,z-1/2. 3,3'-azpy:COOH-Br \#2 x-1,-y+1/2,z+1/2. 3,3'-azpy:OH-I \#2 -x+1/2,y-1/2,-z+3/2. 3,3'azpy:OH-Br \#1 -x+1,y-1/2,-z+1/2 \#2 -x,y+1/2,-z+3/2. 3,3'-azpy:Ox-Br \#1 x,y+1,z+1. 4,4'-azpy:COOH-I \#1 x-2,y+2,z-1. 4,4'-azpy:COOH-Br \#1 x1,y+1,z+1. 4,4'-azpy:OH-I \#1 x-2,y+1,z+1. 4,4'-azpy:OH-Br \#1 x+2,y-1,z-1.4,4'-azpy:Ox-I \#1 x-1/2,-y-1/2,z+1/2. 4,4'-azpy:Ox-Br \#1 x-1/2,-y+3/2,z$51 / 2$.

Table 4a Crystallographic parameters of 3,3'-azobipyridine co-crystals

\begin{tabular}{|c|c|c|c|c|c|c|}
\hline Formula moiety & $\begin{array}{c}\text { 3,3'-azpy:COOH-I } \\
\left(\mathrm{C}_{10} \mathrm{H}_{8} \mathrm{~N}_{4}\right) \\
\left(\mathrm{C}_{7} \mathrm{HF}_{4} \mathrm{IO}_{2}\right)_{2}\end{array}$ & $\begin{array}{c}\text { 3,3'-azpy:COOH-Br } \\
\left(\mathrm{C}_{10} \mathrm{H}_{8} \mathrm{~N}_{4}\right) \\
\left(\mathrm{C}_{7} \mathrm{HBrF}_{4} \mathrm{O}_{2}\right)_{2}\end{array}$ & $\begin{array}{c}\text { 3,3'-azpy:OH-I } \\
\left(\mathrm{C}_{10} \mathrm{H}_{8} \mathrm{~N}_{4}\right) \\
\left(\mathrm{C}_{6} \mathrm{HF}_{4} \mathrm{IO}\right)_{2}\end{array}$ & $\begin{array}{c}\text { 3,3'-azpy:OH-Br } \\
\left(\mathrm{C}_{10} \mathrm{H}_{8} \mathrm{~N}_{4}\right) \\
\left(\mathrm{C}_{6} \mathrm{HBrF}_{4} \mathrm{O}\right)_{2}\end{array}$ & $\begin{array}{c}\text { 3,3'-azpy:Ox-I } \\
\left(\mathrm{C}_{10} \mathrm{H}_{8} \mathrm{~N}_{4}\right) \\
\left(\mathrm{C}_{7} \mathrm{H}_{2} \mathrm{~F}_{4} \mathrm{INO}\right)_{2}\end{array}$ & $\begin{array}{c}\text { 3,3'-azpy:Ox-Br } \\
\left(\mathrm{C}_{10} \mathrm{H}_{8} \mathrm{~N}_{4}\right) \\
\left(\mathrm{C}_{7} \mathrm{H}_{2} \mathrm{BrF}_{4} \mathrm{NO}\right)_{2}\end{array}$ \\
\hline Empirical formula & $\mathrm{C}_{24} \mathrm{H}_{10} \mathrm{~F}_{8} \mathrm{I}_{2} \mathrm{~N}_{4} \mathrm{O}_{4}$ & $\mathrm{C}_{24} \mathrm{H}_{10} \mathrm{Br}_{2} \mathrm{~F}_{8} \mathrm{~N}_{4} \mathrm{O}_{4}$ & $\mathrm{C}_{22} \mathrm{H}_{10} \mathrm{~F}_{8} \mathrm{I}_{2} \mathrm{~N}_{4} \mathrm{O}_{2}$ & $\mathrm{C}_{22} \mathrm{H}_{10} \mathrm{Br}_{2} \mathrm{~F}_{8} \mathrm{~N}_{4} \mathrm{O}_{2}$ & $\mathrm{C}_{24} \mathrm{H}_{12} \mathrm{~F}_{8} \mathrm{I}_{2} \mathrm{~N}_{6} \mathrm{O}_{2}$ & $\mathrm{C}_{24} \mathrm{H}_{12} \mathrm{Br}_{2} \mathrm{~F}_{8} \mathrm{~N}_{6} \mathrm{O}_{2}$ \\
\hline $\begin{array}{l}\text { Molecular weight } \\
\text { Color. Habit }\end{array}$ & $\begin{array}{c}824.16 \\
\text { orange rod }\end{array}$ & 730.18 & 768.14 & 674.16 & $\begin{array}{c}822.20 \\
\text { orange prism }\end{array}$ & 728.22 \\
\hline $\begin{array}{l}\text { Color, Habit } \\
\text { Crystal system }\end{array}$ & $\begin{array}{l}\text { orange rod } \\
\text { Monoclinic }\end{array}$ & $\begin{array}{l}\text { orange plate } \\
\text { Monoclinic }\end{array}$ & $\begin{array}{l}\text { orange plate } \\
\text { Monoclinic }\end{array}$ & orange prism & orange prism & orange plate \\
\hline Space group, Z & $\mathrm{P} 2_{1} / \mathrm{c}, 2$ & $\mathrm{P}_{1} / \mathrm{c}, 2$ & C2/c, 4 & $\mathrm{P} 2_{1} / \mathrm{c}, 4$ & P-1, 1 & $\begin{array}{l}\text { Iriclinic } \\
\mathrm{P}-1,1\end{array}$ \\
\hline $\mathrm{a}, \AA$ & $4.9020(3)$ & $4.8724(3)$ & $19.0228(11)$ & $18.2149(8)$ & $5.6341(4)$ & $8.3500(8)$ \\
\hline $\mathrm{b}, \AA$ & $21.4561(12)$ & $22.0007(13)$ & $16.8636(10)$ & $16.4379(7)$ & $10.4834(7)$ & $8.3646(8)$ \\
\hline $\mathrm{c}, \AA \AA$ & $12.1298(7)$ & $11.6936(7)$ & $7.2425(4)$ & $7.4736(4)$ & $11.5197(8)$ & $9.8996(10)$ \\
\hline$\alpha,{ }^{\circ}$ & 90.00 & 90.00 & 90.00 & 90.00 & $75.365(2)$ & $105.765(3)$ \\
\hline$\beta,{ }^{\circ}$ & $100.151(2)$ & $101.336(3)$ & $93.198(2)$ & $99.631(2)$ & $77.562(2)$ & $103.151(3)$ \\
\hline$\gamma,{ }^{\circ}$ & 90.00 & 90.00 & 90.00 & 90.00 & $80.975(2)$ & $99.733(3)$ \\
\hline Volume, $\AA^{3}$ & $1255.82(13)$ & $1229.06(13)$ & $2319.7(2)$ & $2206.17(18)$ & $639.09(8)$ & $628.00(11)$ \\
\hline X-ray wavelength & 0.71073 & 1.54178 & 0.71073 & 0.71073 & 0.71073 & 0.71073 \\
\hline$\mu, \mathrm{mm}^{-1}$ & 2.606 & 5.151 & 2.806 & 3.774 & 2.556 & 3.325 \\
\hline Absorption corr & multi-scan & multi-scan & multi-scan & multi-scan & multi-scan & multi-scan \\
\hline Trans min / max & $0.4893 / 0.8186$ & $0.5657 / 0.7531$ & $0.4671 / 0.8497$ & $0.3602 / 0.4906$ & $\begin{array}{ll}0.4951 & 0.7161\end{array}$ & $0.4159 / 0.8255$ \\
\hline $\begin{array}{r}\text { Rerlections } \\
\text { collected }\end{array}$ & 13823 & 7259 & 18196 & 62309 & 15760 & 10635 \\
\hline independent & 4126 & 2158 & 3957 & 7628 & 4637 & 3773 \\
\hline observed & 3713 & 2046 & 3666 & 6276 & 4497 & 3174 \\
\hline Threshold expression & $>2 \sigma(\mathrm{I})$ & $>2 \sigma(\mathrm{I})$ & $>2 \sigma(\mathrm{I})$ & $>2 \sigma(\mathrm{I})$ & $>2 \sigma(\mathrm{I})$ & $>2 \sigma(\mathrm{I})$ \\
\hline $\mathrm{R}_{1}$ (observed) & 0.0207 & 0.0261 & 0.0176 & 0.0265 & 0.0186 & 0.0480 \\
\hline$w \mathrm{R}_{2}$ (all) & 0.0508 & 0.0688 & 0.0463 & 0.0699 & 0.0444 & 0.1288 \\
\hline $\mathrm{S}$ & 1.064 & 1.084 & 1.021 & 0.998 & 1.085 & 1.052 \\
\hline
\end{tabular}




\section{Cite this: DOI: 10.1039/coxxooooox}

Table $4 \mathbf{b}$ Crystallographic parameters of 4,4'-azobipyridine co-crystals

\begin{tabular}{|c|c|c|c|c|c|c|}
\hline Formula moiety & $\begin{array}{c}\text { 4,4'-azpy:COOH-I } \\
\left(\mathrm{C}_{10} \mathrm{H}_{8} \mathrm{~N}_{4}\right) \\
\left(\mathrm{C}_{7} \mathrm{HF}_{4} \mathrm{IO}_{2}\right)\end{array}$ & $\begin{array}{c}\text { 4,4'-azpy:COOH-Br } \\
\left(\mathrm{C}_{10} \mathrm{H}_{8} \mathrm{~N}_{4}\right) \\
\left(\mathrm{C}_{7} \mathrm{HBrF}_{4} \mathrm{O}_{2}\right)\end{array}$ & $\begin{array}{c}\text { 4,4'-azpy:OH-I } \\
\left(\mathrm{C}_{10} \mathrm{H}_{8} \mathrm{~N}_{4}\right) \\
\left(\mathrm{C}_{6} \mathrm{HF}_{4} \mathrm{IO}\right)\end{array}$ & $\begin{array}{c}\text { 4,4'-azpy:OH-Br } \\
\left(\mathrm{C}_{10} \mathrm{H}_{8} \mathrm{~N}_{4}\right) \\
\left(\mathrm{C}_{6} \mathrm{HBrF}_{4} \mathrm{O}\right)\end{array}$ & $\begin{array}{c}\text { 4,4'-azpy:Ox-I } \\
\left(\mathrm{C}_{10} \mathrm{H}_{8} \mathrm{~N}_{4}\right) \\
\left(\mathrm{C}_{7} \mathrm{H}_{2} \mathrm{~F}_{4} \mathrm{INO}\right)\end{array}$ & $\begin{array}{c}\text { 4,4'-azpy:Ox-Br } \\
\left(\mathrm{C}_{10} \mathrm{H}_{8} \mathrm{~N}_{4}\right) \\
\left(\mathrm{C}_{7} \mathrm{H}_{2} \mathrm{BrF}_{4} \mathrm{NO}\right)_{2}\end{array}$ \\
\hline Empirical formula & $\mathrm{C}_{17} \mathrm{H}_{9} \mathrm{~F}_{4} \mathrm{IN}_{4} \mathrm{O}_{2}$ & $\mathrm{C}_{17} \mathrm{H}_{9} \mathrm{BrF}_{4} \mathrm{~N}_{4} \mathrm{O}_{2}$ & $\mathrm{C}_{16} \mathrm{H}_{9} \mathrm{~F}_{4} \mathrm{IN}_{4} \mathrm{O}$ & $\mathrm{C}_{16} \mathrm{H}_{9} \mathrm{BrF}_{4} \mathrm{~N}_{4} \mathrm{O}$ & $\mathrm{C}_{17} \mathrm{H}_{10} \mathrm{~F}_{4} \mathrm{IN}_{5} \mathrm{O}$ & $\mathrm{C}_{24} \mathrm{H}_{12} \mathrm{Br}_{2} \mathrm{~F}_{8} \mathrm{~N}_{6} \mathrm{O}_{2}$ \\
\hline Molecular weight & 504.18 & 457.19 & 476.17 & 429.18 & 503.20 & 728.22 \\
\hline Color, Habit & bronze plate & orange prism & orange plate & colourless plate & orange plate & orange needle \\
\hline Crystal system & Triclinic & Triclinic & Triclinic & Triclinic & Monoclinic & Monoclinic \\
\hline Space group, $\mathrm{Z}$ & $\mathrm{P} 1,1$ & $\mathrm{P}-1,4$ & $\mathrm{P}-1,2$ & $\mathrm{P}-1,2$ & $\mathrm{C} 2 / \mathrm{c}, 8$ & $\mathrm{P} 2_{1} / \mathrm{n}, 2$ \\
\hline $\mathrm{a}, \AA$ & $6.2112(8)$ & $10.1607(5)$ & $6.1515(5)$ & $6.3706(3)$ & $17.6282(7)$ & $10.2846(10)$ \\
\hline $\mathrm{b}, \AA$ & $8.1879(11)$ & $13.4553(6)$ & $9.8197(7)$ & $9.5670(5)$ & $6.3167(3)$ & $4.6215(5)$ \\
\hline $\mathrm{c}, \AA$ & $8.9824(12)$ & $14.4474(8)$ & $13.7443(10)$ & $13.3289(7)$ & $31.7318(14)$ & $27.841(2)$ \\
\hline$\alpha,{ }^{\circ}$ & $84.843(3)$ & $112.158(3)$ & $81.558(3)$ & $79.453(2)$ & 90.00 & 90.00 \\
\hline$\beta,^{\circ}$ & $70.837(3)$ & $90.592(3)$ & $85.932(3)$ & $83.090(2)$ & $96.518(2)$ & $98.246(6)$ \\
\hline$\gamma, \circ$ & $77.402(3)$ & $111.504(3)$ & $78.342(3)$ & $79.4760(10)$ & 90.00 & 90.00 \\
\hline Volume, $\AA^{3}$ & $421.03(10)$ & $1676.48(14)$ & $803.57(10)$ & $782.03(7)$ & $3510.6(3)$ & $1309.6(2)$ \\
\hline $\mathrm{X}$-ray wavelength & 0.71073 & 1.54178 & 1.54178 & 0.71073 & 0.71073 & 1.54178 \\
\hline$\mu, \mathrm{mm}^{-1}$ & 1.967 & 3.967 & 16.211 & 2.687 & 1.884 & 4.795 \\
\hline Absorption corr & multi-scan & multi-scan & multi-scan & multi-scan & multi-scan & multi-scan \\
\hline Trans $\min / \max$ & $0.5544 / 0.9255$ & $0.5354 / 0.7420$ & $0.1813 / 0.5633$ & $0.5804 / 0.7464$ & $0.6401 / 0.9284$ & $0.5662 / 0.7531$ \\
\hline Reflections & & & & & & \\
\hline collected & 8655 & 18301 & 11713 & 17166 & 27187 & 7830 \\
\hline independent & 4606 & 5840 & 2764 & 5315 & 6206 & 2257 \\
\hline observed & 4415 & 5035 & 2604 & 4829 & 5308 & 1721 \\
\hline Threshold expression & $>2 \sigma(\mathrm{I})$ & $>2 \sigma(\mathrm{I})$ & $>2 \sigma(\mathrm{I})$ & $>2 \sigma(\mathrm{I})$ & $>2 \sigma(\mathrm{I})$ & $>2 \sigma(\mathrm{I})$ \\
\hline $\mathrm{R}_{1}$ (observed) & 0.0433 & 0.0851 & 0.0275 & 0.0269 & 0.0239 & 0.0595 \\
\hline$w \mathrm{R}_{2}$ (all) & 0.1149 & 0.2211 & 0.0756 & 0.0720 & 0.0575 & 0.1586 \\
\hline $\mathrm{S}$ & 1.062 & 1.038 & 1.082 & 1.037 & 1.040 & 1.099 \\
\hline
\end{tabular}

\section{Results}

\section{Co-crystals of 3,3'-azobipyridine}

3,3'-Azobipyridine:4-iodotetrafluorobenzoic acid The crystal structure determination of 3,3'-azpy:COOH-I

10 shows that the result is a neutral molecular solid with two acid molecules for every one bipy. The co-crystal is formed via two symmetry related $\mathrm{O}-\mathrm{H} \cdots \mathrm{N}$ hydrogen bonds between one carboxylic acid and a nitrogen atom, Table 2. These interactions lead to trimeric supermolecules which are 15 subsequently connected into 2-D corrugated sheets via C$\mathrm{I} \cdots \mathrm{O}_{(\mathrm{C}=\mathrm{O})}$, (Fig 1).

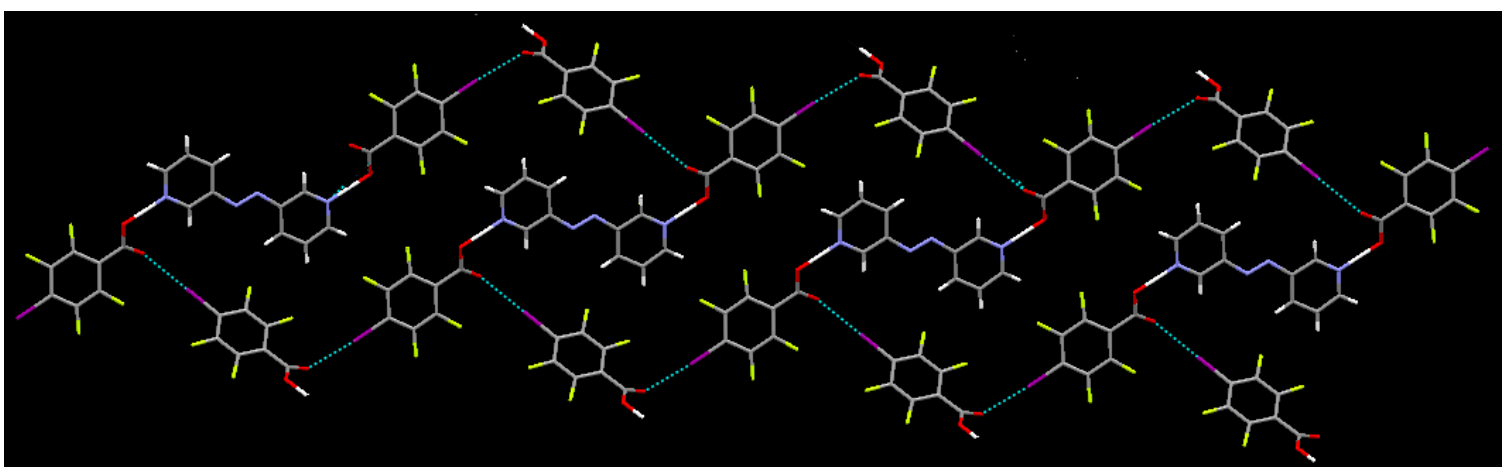

Fig.1 2-D network formed via strong $\mathrm{O}-\mathrm{H} \cdots \mathrm{N}_{(\mathrm{py})}$ and weaker $\mathrm{C}-\mathrm{I} \cdots \mathrm{O}_{(\mathrm{C}=\mathrm{O})}$ interactions in 3,3'-azpy:COOH-I co-crystal. The apparent 'covalent' bond between $\mathrm{COOH}$ and py is due to a disorder over two positions of the haloacid.

3,3’-Azobipyridine:4-bromotetrafluorobenzoic acid 
In 3,3'-azpy: $\mathbf{C O O H}-\mathbf{B r}$ co-crystal, the primary driving force is the acid-pyridine $\mathrm{O}-\mathrm{H} \cdots \mathrm{N}$ hydrogen bonds, with two acid molecules for every single bipyridine. The neutral trimers thus formed extend further into 2-D sheets via $\mathrm{C}$ ${ }_{5} \mathrm{Br}_{\cdots} \mathrm{O}_{(\mathrm{C}=\mathrm{O})}$ interactions (Fig 2).

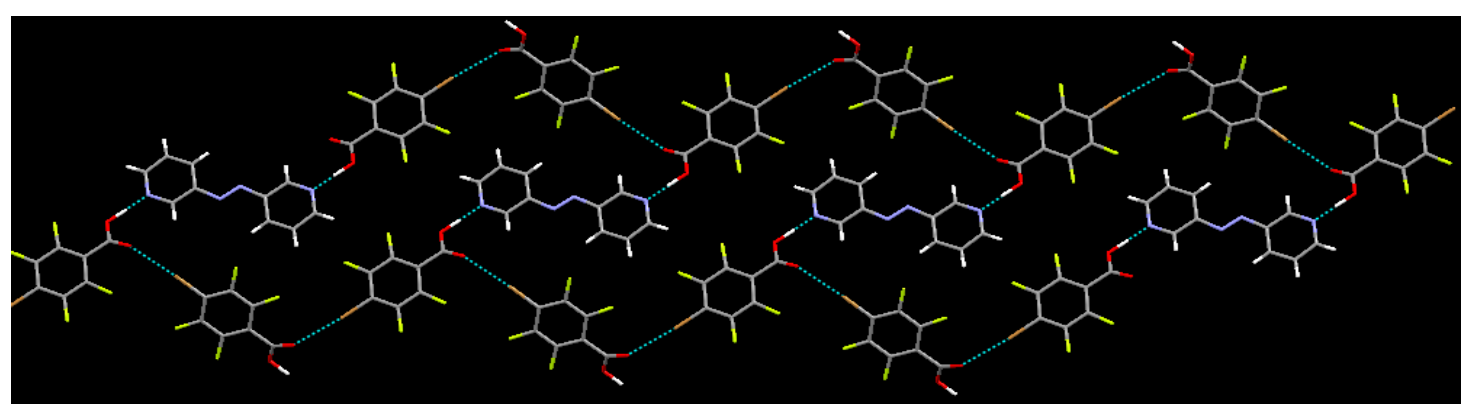

Fig. 2 3,3'-azpy: $\mathrm{COOH}-\mathrm{Br}$ co-crystal showing primary $\mathrm{O}-\mathrm{H} \cdots \mathrm{N}_{(\mathrm{py})}$ and secondary $\mathrm{C}-\mathrm{Br} \cdots \mathrm{O}_{(\mathrm{C}=\mathrm{O})}$ interactions

\section{3,3'-Azobipyridine:4-iodotetrafluorophenol}

4-Iodotetrafluorophenol has two potential single point donors, i.e. I and $\mathrm{OH}$ that could potentially bind to the nitrogen atoms. In 3,3'-azpy:OH-I however, hydroxyl proton interacts with the nitrogen atoms via symmetry related $\mathrm{O}-\mathrm{H} \cdots \mathrm{N}$ hydrogen
15 bonds leading to a trimeric supermolecule. These trimers are then further connected along 2-D sheets via weaker C$\mathrm{I} \cdots \mathrm{O}_{(\mathrm{C}=\mathrm{O})}$ interactions (Fig 3)

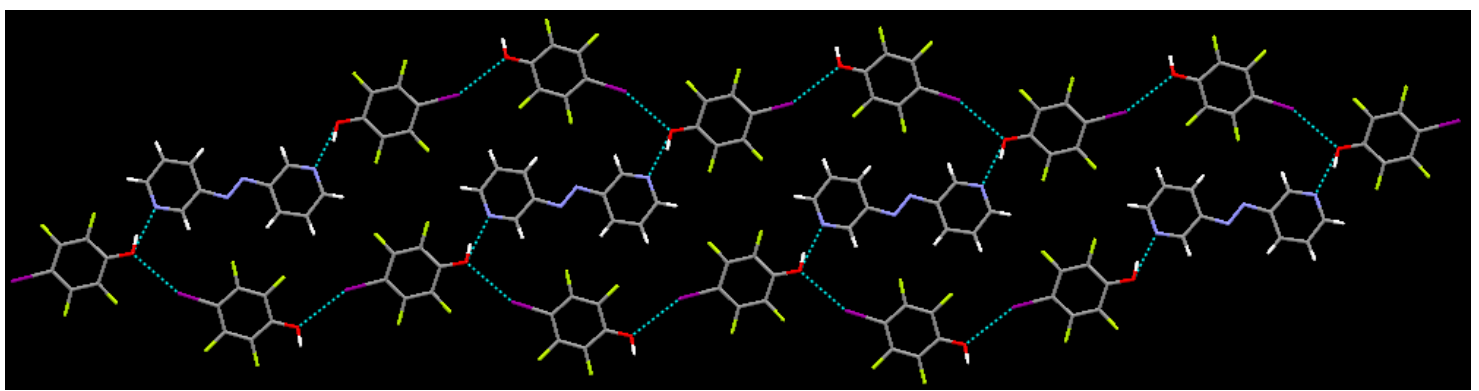

Fig.3 3,3'-azpy:OH-I crystal structure showing primary $\mathrm{O}-\mathrm{H} \cdots \mathrm{N}_{(\mathrm{py})}$ and secondary $\mathrm{C}-\mathrm{I} \cdots \mathrm{O}_{(\mathrm{C}=\mathrm{O})}$ interactions forming an eight component repeat unit

20

3,3'-Azobipyridine:4-Bromotetrafluorophenol

In 3,3'-azpy:OH-Br co-crystal, intermolecular $\mathrm{O}-\mathrm{H} \cdots \mathrm{N}$

hydrogen bonds direct the formation of primary trimeric building blocks. The interaction of bromine atom with 25 hydroxyl oxygen lone pairs via $\mathrm{C}-\mathrm{Br} \cdots \mathrm{O}$ bond connects these building blocks in two dimensions (Fig 4).

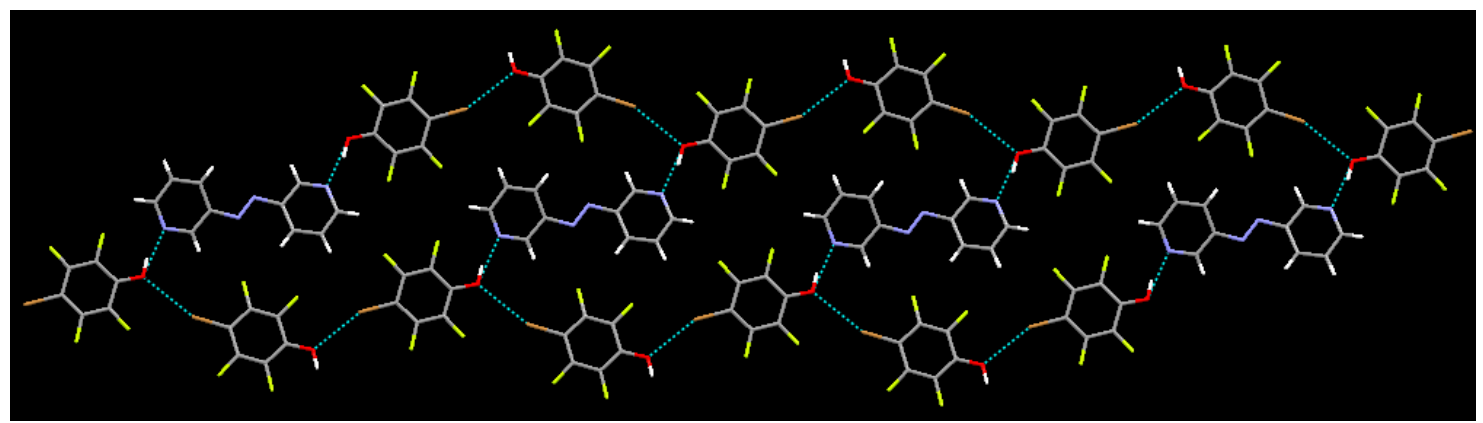

Fig.4 3,3'-azpy:OH-Br crystal structure showing intermolecular $\mathrm{O}-\mathrm{H} \cdots \mathrm{N}_{(\mathrm{py})}$ and $\mathrm{C}-\mathrm{Br} \cdots \mathrm{O}$ interactions forming the multi-component supermolecule

\section{3,3'-Azobipyridine:4-iodotetrafluoroaldoxime}

In the co-crystal of 3,3'-azpy-Ox-I, Iodine forms C-I $\cdots \mathrm{N}$ halogen bonds with the two pyridine nitrogen atoms, while the oxime engages itself in a dimer formation with another donor molecule (Fig 5). This results in 1-D non-planar corrugated 35 chain like assembly with the donor and acceptor molecules aligned almost perpendicular to each other. Among all the cocrystals of 3,3'-azobipyridine with bifunctional donor molecules, this is the only example in which halogen bonds are formed as the primary mode of interaction, in contrast to 40 hydrogen bonds. 


\section{Journal Name}

\section{Cite this: DOI: 10.1039/coxxooooox}

PAPER

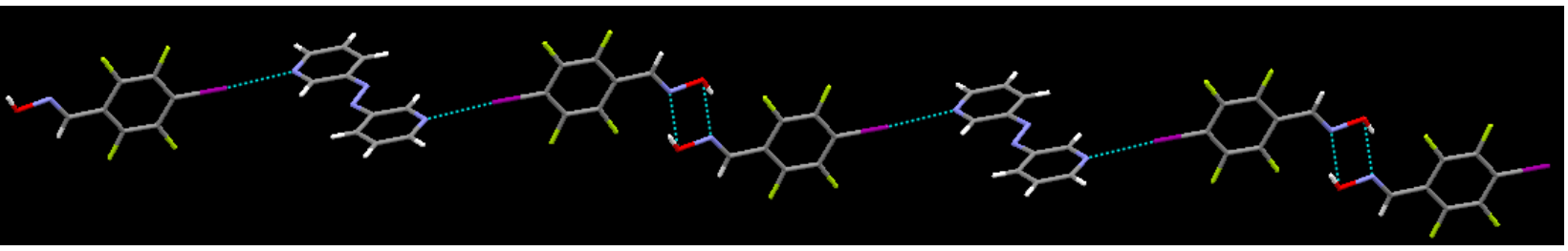

Fig.5 3,3'-azpy:Ox-I co-crystal with oxime-oxime dimer and C-I $\cdots \mathrm{N}_{(\mathrm{py})}$ halogen bonds forming 1-D non-planar corrugated chain

3,3'-Azobipyridine:4-bromotetrafluoroaldoxime

3,3'-azpy:Ox-Br co-crystal shows an aldoxime proton interacting with pyridine via symmetry related $\mathrm{O}-\mathrm{H} \cdots \mathrm{N}$ hydrogen bonds, while the aldoxime oxygen interacts with
10 bromine via $\mathrm{C}-\mathrm{Br} \cdots \mathrm{O}$ contacts forming the supermolecule (Fig $6)$.

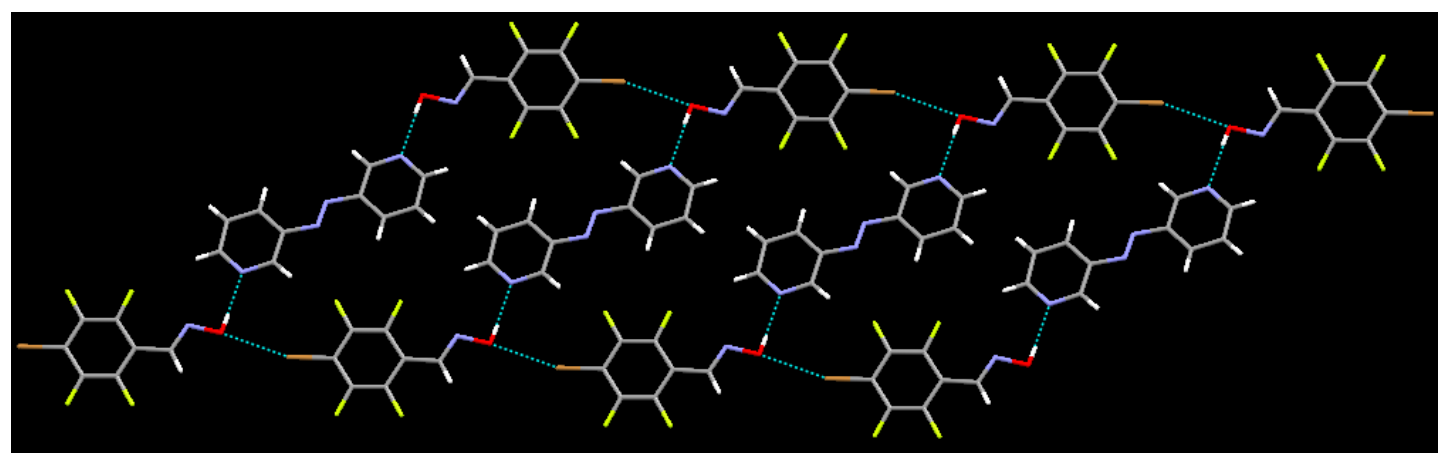

Fig.6 3,3'-azpy:Ox-Br crystal structure showing $\mathrm{O}-\mathrm{H} \cdots \mathrm{N}_{(\mathrm{py})}$ primary hydrogen bonds and secondary $\mathrm{C}-\mathrm{Br} \cdots \mathrm{O}$ interactions 15

\section{Co-crystals of 4,4'-azobipyridine}

20 4,4'-Azobipyridine:4-iodotetrafluorobenzoic acid In 4,4'-azpy:COOH-I co-crystal, intermolecular $\mathrm{O}-\mathrm{H} \cdots \mathrm{N}$ hydrogen bonds as well as $\mathrm{C}-\mathrm{I} \cdots \mathrm{N}$ halogen bond interactions form neutral molecular solids with infinite 1-D chain like architecture (Fig 7).

25

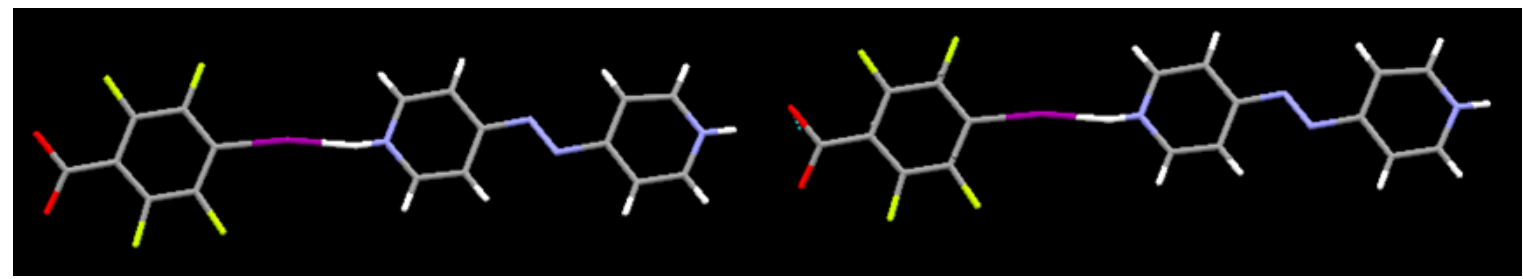

Fig.7 1-D infinite chain in 4,4'-azpy:COOH-I co-crystal formed via $\mathrm{O}-\mathrm{H} \cdots \mathrm{N}_{(\mathrm{py})}$ and $\mathrm{C}-\mathrm{I} \cdots \mathrm{N}_{(\mathrm{py})}$ hydrogen and halogen bond, respectively. For specific information about the crystallography, see the experimental section

30 4,4'-Azobipyridine:4-bromotetrafluorobenzoic acid 4,4'-azpy:COOH-Br co-crystal exhibits infinite 1-D chains formed via intermolecular neutral $\mathrm{O}-\mathrm{H} \cdots \mathrm{N}$ hydrogen bonds as well as $\mathrm{C}-\mathrm{Br} \cdots \mathrm{N}$ halogen bonds on the other (Fig 8). 


\section{Cite this: DOI: 10.1039/coxxooooox}

PAPER

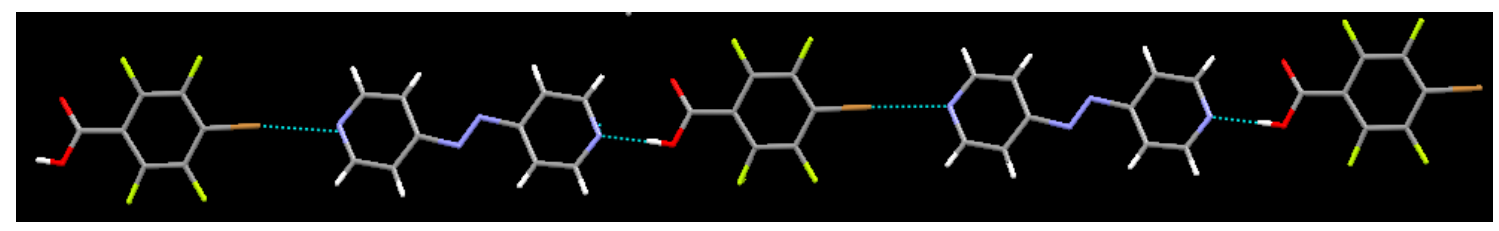

Fig.8 4,4'-azpy:COOH-Br co-crystal showing primary $\mathrm{O}-\mathrm{H} \cdots \mathrm{N}_{(\mathrm{py})}$ hydrogen bonds and $\mathrm{C}-\mathrm{Br} \cdots \mathrm{N}_{(\mathrm{py})}$ halogen bond interactions

4,4'-Azobipyridine:4-iodotetrafluorophenol

5 In 4,4'-azpy:OH-I co-crystal, both hydroxyl proton and

C-I $\cdots \mathrm{N}_{(\mathrm{py})}$ bonds forming an infinite 1-D chain (Fig 9).

iodine bind to the pyridine $\mathrm{N}$ on either sides, via $\mathrm{O}-\mathrm{H} \cdots \mathrm{N}$ and

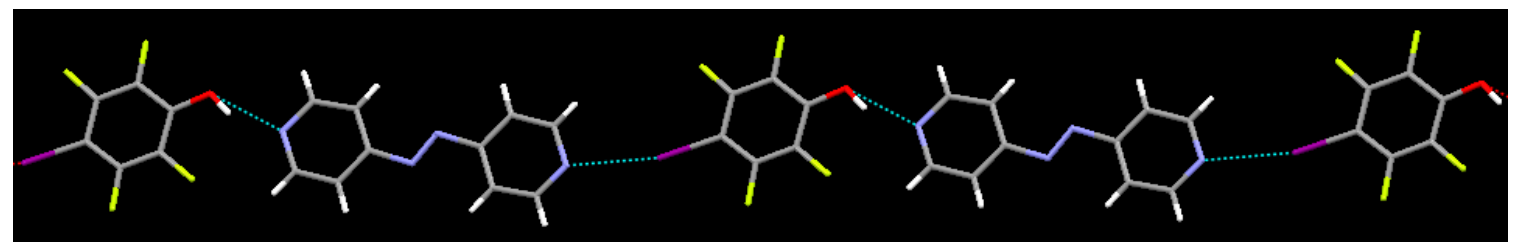

10

Fig.9 4,4'-azpy:OH-I crystal structure indicating 1-D chains formed via both $\mathrm{O}-\mathrm{H} \cdots \mathrm{N}_{(\mathrm{py})}$ and $\mathrm{C}-\mathrm{I} \cdots \mathrm{N}_{(\mathrm{py})}$ primary interactions

4,4'-Azobipyridine:4-bromotetrafluorophenol

4,4'-azpy: $\mathrm{OH}-\mathrm{Br}$ co-crystal displays $1-\mathrm{D}$ infinite chain formed

15 halogen bonds (Fig 10).

via intermolecular $\mathrm{O}-\mathrm{H} \cdots \mathrm{N}$ hydrogen bonds as well as $\mathrm{C}-\mathrm{Br} \cdots \mathrm{N}$

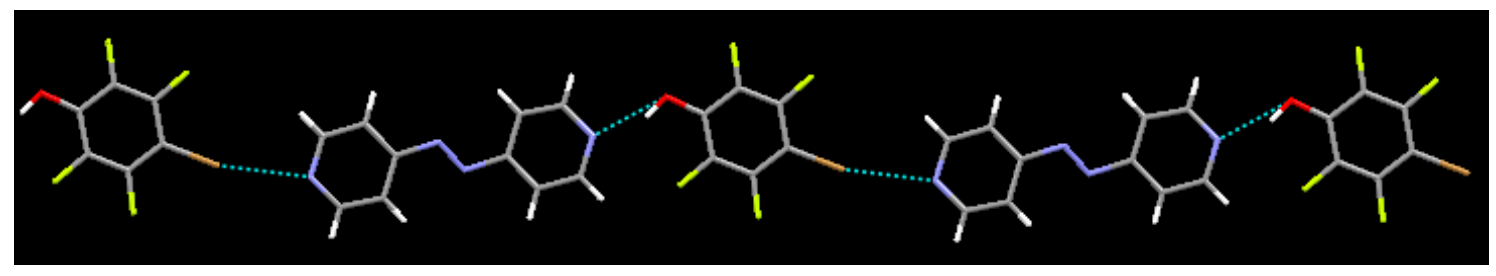

Fig.10 4,4'-azpy:OH-Br co-crystal structure showing 1-D chain formed via both hydrogen $\left(\mathrm{O}-\mathrm{H} \cdots \mathrm{N}_{(\mathrm{py})}\right)$ and halogen $\left(\mathrm{C}-\mathrm{Br} \cdots \mathrm{N}_{(\mathrm{py})}\right)$ bonds

4,4'-Azobipyridine:4-iodotetrafluoroaldoxime

In 4,4'-azpy:Ox-I co-crystal, 1-D infinite zig-zag chains are $(\mathrm{C}-\mathrm{I} \cdots \mathrm{N})$ bonds (Fig 11).

20 formed via both hydrogen $(\mathrm{O}-\mathrm{H} \cdots \mathrm{N})$ as well as halogen bonds

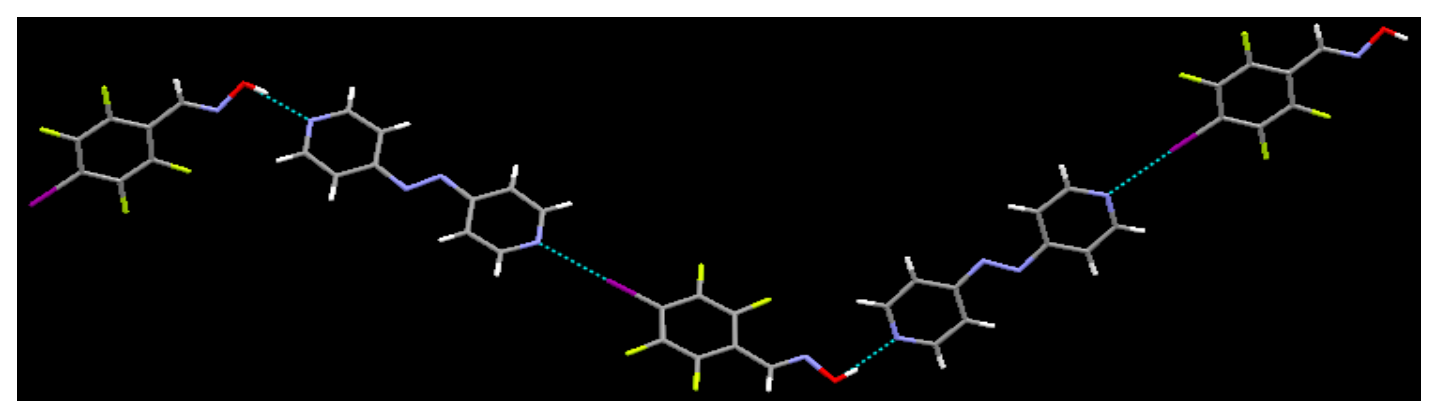

Fig.11 4,4'-azpy:Ox-I crystal structure with primary $\mathrm{C}-\mathrm{I} \cdots \mathrm{N}_{(\mathrm{py})}$ and $\mathrm{O}-\mathrm{H} \cdots \mathrm{N}_{(\mathrm{py})}$ interactions forming 1-D tape 


\section{4,4'-Azobipyridine:4-bromotetrafluoroaldoxime}

Unlike in the previous structures, in the 4,4'-azpy:Ox-Br cocrystal, the aldoxime proton interacts via oxime $\cdots$ pyridine hydrogen bonds on both sides, while bromine on the other end 5 displays interactions with the azo nitrogen $\left(\mathrm{C}-\mathrm{Br} \cdots \mathrm{N}_{(\mathrm{azo})}, 3.397 \AA\right)$ (Fig 12a). The bond distance is longer than the normal $\mathrm{C}$ $\mathrm{X} \cdots \mathrm{N}_{\text {aromatic }}(2.97 \AA)$, but slightly shorter than the sum of their van der Waal radii (3.40 $\AA$ ). The halogen bonding ability of azonitrogen is a rare occurrence and does not have many precedents 10 in literature. The $\mathrm{Br} \cdots \mathrm{N}_{(\text {azo }}$ interaction aligns the azobipyridine almost perpendicular to the plane, thus forming 3-D architecture as seen in Fig 12b.

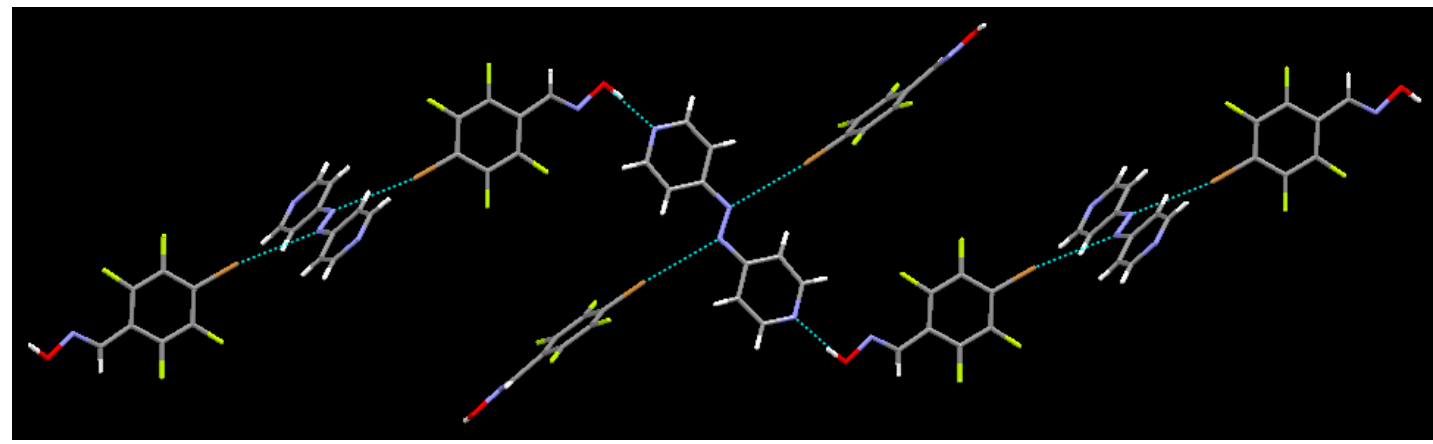

Fig.12a 4,4'-azpy:Ox-Br crystal structure with primary $\mathrm{O}-\mathrm{H} \cdots \mathrm{N}_{(\text {py) }}$ hydrogen bonds and secondary C-Br $\cdots \mathrm{N}_{(\text {azo })}$ interactions

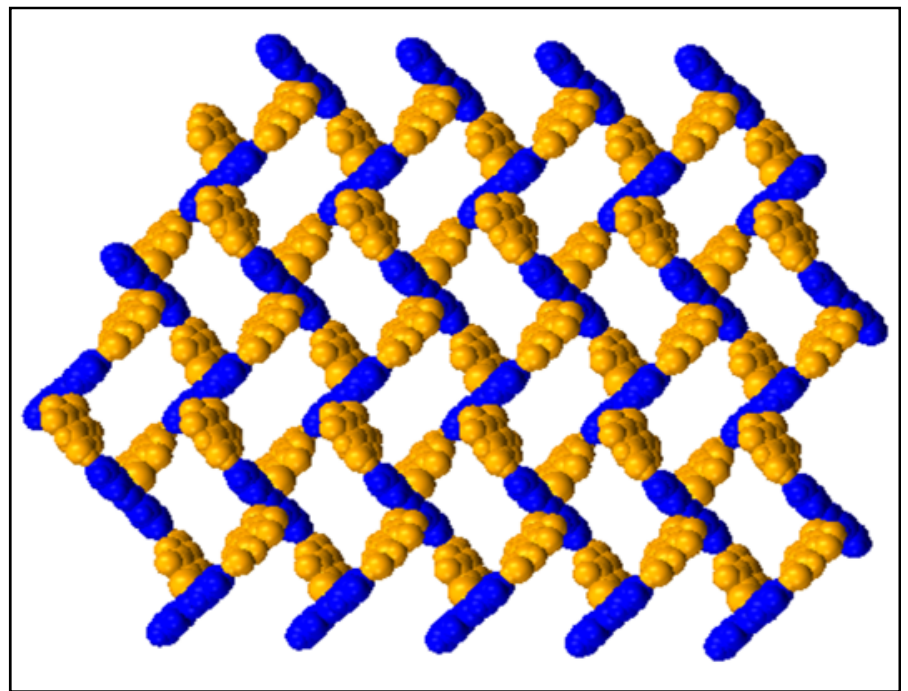

Fig. 12b 3D-architecture in 4,4'azpy:OxBr co-crystal

\section{${ }_{20}$ Discussion}

Twelve co-crystallizations were set up using two isomeric pyridine-based acceptors (3,3'-and 4,4'-azobipyridine) and six bifunctional donors comprising both a $\mathrm{HB}$ donor (acid, phenol and oxime) as well as an XB donor (iodine and bromine) on the same 25 backbone. Single-crystal structural data were acquired for solids obtained from all 12 reactions. The primary interactions driving the crystal assembly were the $\mathrm{O}-\mathrm{H} \cdots \mathrm{N}_{(\mathrm{py})}$ hydrogen bonds or $\mathrm{C}$ $\mathrm{X} \cdots \mathrm{N}_{(\mathrm{py})}$ halogen bonds followed by secondary interactions which were used to connect the primary motifs. In all co-crystals, there 30 were several potential acceptors e.g. $\mathrm{N}(\mathrm{py}), \mathrm{N}(\mathrm{azo}), \mathrm{O}(-\mathrm{O}=\mathrm{C})$ and $\mathrm{O}(-\mathrm{OH})$, and donors e.g. acid, phenol, oxime, iodine and bromine. Considering the complexity of such a system, although it was reasonable to expect some degree of structural 'chaos' in the supramolecular outcome, we observe remarkable consistency in
35 the binding pattern within the two classes of acceptors. Table 5 and Scheme 4 summarize the structural landscape in these twelve crystal structures. 
Table 5 Summary of the structural outcome in 3,3'-and 4,4'azobipyridine co-crystals of dicarboxylic acids.

\begin{tabular}{|c|c|c|c|c|c|c|}
\hline & $\begin{array}{l}\text { COOH-I } \\
\text { (acid-H) }\end{array}$ & $\begin{array}{c}\mathrm{COOH}-\mathrm{Br} \\
\text { (acid-H) }\end{array}$ & $\begin{array}{c}\text { OH-I } \\
\text { (phenol-H) }\end{array}$ & $\begin{array}{c}\mathrm{OH}-\mathrm{Br} \\
\text { (phenol-H) }\end{array}$ & $\begin{array}{c}\text { Ox-Br } \\
\text { (oxime-H) }\end{array}$ & $\begin{array}{c}\text { Ox-I } \\
\text { (oxime-H) }\end{array}$ \\
\hline \multirow{2}{*}{$\begin{array}{l}3,3 \text { '- } \\
\text { azpy }\end{array}$} & $\mathrm{OH}>\mathrm{I}$ & $\mathrm{OH}>\mathrm{Br}$ & $\mathrm{OH}>\mathrm{I}$ & $\mathrm{OH}>\mathrm{Br}$ & $\mathrm{OH}_{\mathrm{ox}}>\mathrm{Br}$ & $\mathrm{OH}_{\mathrm{ox}}<\mathrm{I}$ \\
\hline & A & A & $\mathbf{A}$ & $\mathbf{A}$ & $\mathbf{A}$ & C \\
\hline \multirow[t]{2}{*}{$\begin{array}{l}4,4 \text { '- } \\
\text { azpy }\end{array}$} & $\mathrm{OH}=\mathrm{I}$ & $\mathrm{OH}=\mathrm{Br}$ & $\mathrm{OH}=\mathrm{I}$ & $\mathrm{OH}=\mathrm{Br}$ & $\mathrm{OH}_{\mathrm{ox}}>\mathrm{Br}$ & $\mathrm{OH}_{\mathrm{ox}}=\mathrm{I}$ \\
\hline & B & B & B & B & $\mathbf{A}$ & B \\
\hline
\end{tabular}

\section{3,3'-azopyridine co-crystals}
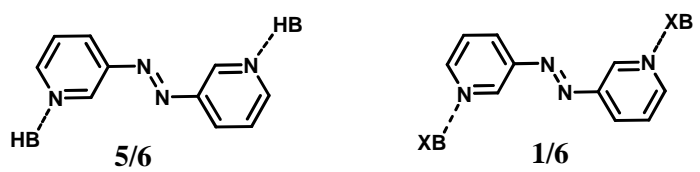

\section{4,4'-azopyridine co-crystals}

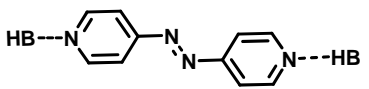

$1 / 6$

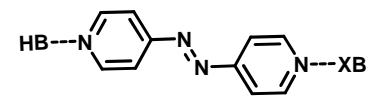

$5 / 6$
Scheme 4 Summary of the outcome in 3,3'-and 4,4'-azobipyridine cocrystals.

Cambridge Structural Database (CSD) was used to compare the intermolecular bond distances for both hydrogen $(\mathrm{O}-\mathrm{H} \cdots \mathrm{N})$ as well as halogen bonds $(\mathrm{C}-\mathrm{X} \cdots \mathrm{N})$. The search in CSD was limited 10 to aromatics alone. The average intermolecular hydrogen bond distances reported for acid-pyridine, phenol-pyridine and aldoxime-pyridine are $1.749 \AA, 1.935 \AA$ and $1.984 \AA$ respectively; while for our crystal structures the corresponding values are 1.66 $\AA, 1.79 \AA$ and $1.88 \AA$. There is a good agreement in the trend 15 observed for the $\mathrm{O}-\mathrm{H} \cdots \mathrm{N}$ bond distances in the reported as well as observed values which decreases in the following order, oximepyridine $>$ phenol-pyridine $>$ acid-pyridine.

Similar search on aromatic halogen-pyridine halogen bond distance gives an average C-I $\cdots \mathrm{N}$ and $\mathrm{C}-\mathrm{Br} \cdots \mathrm{N}$ value of $2.883 \AA$ 20 and $3.039 \AA$ respectively. Shorter bond distance for $\mathrm{I} \cdots \mathrm{N}$ interaction signifies that iodine is a better donor than bromine. Based on our crystal structures, the average C-I $\cdots \mathrm{N}$ and $\mathrm{C}-\mathrm{Br} \cdots \mathrm{N}$ bond distance is $2.850 \AA$ and $2.895 \AA$ respectively, which again is in good agreement with the results from the database and the 25 electrostatic argument. Moreover, iodine forms C- $\mathrm{I} \cdots \mathrm{N}_{(\mathrm{py})}$ primary halogen bonds with the most basic pyridine nitrogen in $4 / 6$ cases, whereas bromine forms $\mathrm{C}-\mathrm{Br} \cdots \mathrm{N}_{(\mathrm{py})}$ halogen bond in only $2 / 6$ instances. Bromine interacts more frequently with less basic secondary acceptors, i.e. ' $\mathrm{O}$ ' and ' $\mathrm{N}_{\text {azo }}$ ' 4/6 times, whereas iodine 30 does so only $2 / 6$ times.

Five out of six 3,3'-azobipyridine co-crystals (COOH-I/COOH$\mathrm{Br} / \mathrm{OH}-\mathrm{I} / \mathrm{OH}-\mathrm{Br} / \mathrm{Ox}-\mathrm{Br}$ ) exhibit $\mathrm{OH} \cdots \mathrm{N}_{(\mathrm{py})}$ interactions involving the best acceptor leading to supramolecular trimers. The trimers
35 are then linked into 2-D corrugated sheets via weaker interactions between $\mathrm{I} / \mathrm{Br}$ with a secondary acceptor such as an oxygen atom. Thus, in this group of structures, hydrogen bonds dominate over halogen bonds, i.e. $\mathrm{HB}>\mathrm{XB}(5 / 6)$ (Scheme 4). An outlier in this series is 3,3'-azpy:Ox-I, where the $\mathrm{XB}$ donor interacts 40 preferentially with the $\mathrm{N}_{(\mathrm{py})}$ acceptor. As a result, the 'free' oxime moiety forms a dimer with an oxime from a neighbouring molecule leading to infinite 1-D chain-like structure. ${ }^{24}$ In this crystal structure, since the hydrogen-bonding group (oxime) is involved in a homomeric dimer, the halogen bond 'wins', i.e. HB ${ }_{45}<\mathrm{XB}(1 / 6)$ (Scheme 4).

In contrast, in five out of six 4,4'-azobipyridine co-crystals (COOH-I/COOH-Br/OH-I/OH-Br/Ox-I) 1-D chains are obtained via both $\mathrm{O}-\mathrm{H} \cdots \mathrm{N}_{(\mathrm{py})}$ hydrogen and $\mathrm{C}-\mathrm{X} \cdots \mathrm{N}_{(\mathrm{py})}(\mathrm{X}=\mathrm{I} / \mathrm{Br})$ halogen bonds with the $\mathrm{N}(\mathrm{py})$ acceptor site. In this group, both donors are 50 of equal importance in the primary assembly process, i.e. $\mathrm{HB} \cong$ $\mathrm{XB}(5 / 6)$ (Scheme 4). There is no distinction between bromine and iodine as a halogen-bond donor in any of the co-crystals. An outlier in the series is 4,4'-azpy:Ox-Br where the oxime $\cdots$ pyridine hydrogen bonds direct the crystal structure i.e. $\mathrm{HB}>\mathrm{XB}(1 / 6)$ 55 (Scheme 4). It is interesting to note the appearance of a rare $\mathrm{C}$ $\mathrm{Br} \cdots \mathrm{N}_{(\mathrm{azo})}$ halogen bond in this structure. The azo nitrogen is a very weak acceptor compared to oxygen and hence the inclination of bromine to bind to it is surprising. This interaction aligns the azobipyridine almost perpendicular to the plane thus leading to a

60 three dimensional molecular assembly. This observation does introduce the possibility for a new prospective acceptor site (azo nitrogen) that could be further explored in designing new strategies for directed assembly of complex supramolecular architectures.

${ }_{65}$ There is a clear distinction between the primary molecular recognition events in the two classes of azobipyridine co-crystals with same co-formers. In one case, hydrogen bonding is the preferred interaction whereas in the other case $\mathrm{HB}$ and $\mathrm{XB}$ seem to be of equal importance. This difference cannot be explained on 70 the basis of any charge argument on the acceptor molecules, since the charges on the nitrogen atoms in the two azobipyridines differ by only $2 \mathrm{~kJ} / \mathrm{mol}$. In order to seek a plausible explanation for the difference in binding behavior we examined the electrostatic potentials of donor molecules as well as packing coefficients and 75 melting point trends in the two classes of co-crystals.

\section{Electrostatic surface potential calculations}

Geometry optimization and surface potential calculations were performed on six bi-functional donor molecules using Spartan, ${ }_{80} 2010$ (Wave function, Inc. Irvine, CA). Calculations were done using both Semi-empirical (PM3) as well as Density Functional Theory (DFT), (B3LYP, 6-31++G** basis set) models. Table 6 summarizes the outcome of theoretical calculations.

Table 6 Electrostatic surface potentials expressed as charges in $\mathrm{kJ} / \mathrm{mol}$

\begin{tabular}{cc|cc|cc|cc|cc|cc|c}
\hline \multicolumn{1}{c}{ COOH-I } & COOH-Br & \multicolumn{1}{c}{ OH-I } & \multicolumn{1}{c}{ OH-Br } & \multicolumn{2}{c}{ Ox-I } & \multicolumn{2}{c}{ Ox-Br } \\
& H & I & H & Br & H & I & H & Br & H & I & H & Br \\
PM3 & 144 & 222 & 144 & 160 & 156 & 202 & 163 & 152 & 134 & 204 & 135 & 147 \\
DFT & 296 & 166 & 305 & 142 & 305 & 155 & 314 & 121 & 283 & 153 & 276 & 129
\end{tabular}

85

Based on both PM3 as well as DFT calculations, the following 
trend in surface potentials is observed among the hydrogen bond donors, phenol $>$ acid $>$ aldoxime, while the among the halogen atoms iodine shows a greater positive sigma hole potential compared to bromine. However; when comparing the relative 5 sigma potentials of hydrogen atom and halogen atom on the same molecule, PM3 calculations show a higher positive potential for halogen atom whereas DFT shows a higher potential for hydrogen atom. The electrostatic potential values do not show any apparent differences or trends between hydrogen and halogen 10 bond atom that could explain the two different molecular recognition events witnessed in 3,3'- and 4,4'- azpy co-crystals, and hence could not be used to corroborate the experimental trend.

\section{${ }_{15}$ Packing Coefficients and Melting points}

Packing coefficients were calculated for all 12 co-crystals using the Olex 2 v1.2 software. Table $7 \mathrm{a}$ and $7 \mathrm{~b}$ summarizes the packing coefficient and melting point values of all 3,3'-azpy and 4,4'azpy co-crystals

20 Table 7a 3,3'-azpy cocrystals

\begin{tabular}{|c|c|c|c|c|c|c|}
\hline & $\mathrm{COOH}-\mathrm{I}$ & $\mathrm{COOH}-\mathrm{Br}$ & OH-I & $\mathrm{OH}-\mathrm{Br}$ & Ox-I & $\mathrm{Ox}-\mathrm{Br}$ \\
\hline $\begin{array}{l}\text { Packing } \\
\text { coefficient } \\
(\%)\end{array}$ & 74 & 69 & 68 & 71 & 69 & 69 \\
\hline $\begin{array}{l}\text { Melting } \\
\text { points } \\
\left({ }^{\circ} \mathrm{C}\right)\end{array}$ & $182-185$ & $155-157$ & $125-127$ & $170-173$ & $145-148$ & $105-108$ \\
\hline
\end{tabular}

Table 7b 4,4'-azpy co-crystals

\begin{tabular}{|c|c|c|c|c|c|c|}
\hline & COOH-I & $\mathrm{COOH}-\mathrm{Br}$ & OH-I & $\mathrm{OH}-\mathrm{Br}$ & Ox-I & $\mathrm{Ox}-\mathrm{Br}$ \\
\hline $\begin{array}{c}\text { Packing } \\
\text { coefficient } \\
(\%)\end{array}$ & 74 & 76 & 67 & 69 & 66 & 67 \\
\hline $\begin{array}{l}\text { Melting } \\
\text { points } \\
\left({ }^{\circ} \mathrm{C}\right)\end{array}$ & $220-222$ & 190-191 & $163-165$ & $133-136$ & $115-116$ & $125-126$ \\
\hline
\end{tabular}

The packing coefficients for the two classes of co-crystals range 25 from $66-74 \%$ and they show very similar values with the same donor molecule. Since no obvious differences are observed in the packing coefficient values in 3,3'- and 4,4'-azpy classes of cocrystals it could not be used to validate the observed differences in supramolecular architectures. Melting points of co-crystals 30 show a wide range and also fail to show any convincing trend to be able to explain the differences in the molecular assemblies formed. Also no clear correlation is observed between the packing coefficients and melting points of a given co-crystal.

${ }_{35}$ Since theoretical calculations, packing coefficients and melting points could not be used to explain the differences in binding events, this 'synthon crossover', ${ }^{25}$ could be rationalized based on the difference in binding site orientation in the two azobipyridine molecules.

${ }_{40}$ The nitrogen atoms on 3,3'-azobipyridine have an anti-parallel orientation with respect to each other (Scheme 1). This geometry does not favour the ready formation of 1-D chains as the lack of ideal complementary means that adjacent molecules need to be forcefully brought in close proximity at angles that can enforce a 45 1-D assembly formation. In doing so the overall energy of the system may increase while its stability is compromised. On the other hand, forming individual trimers is less challenging and comes natural to the system. These trimers can then be readily connected via secondary interactions thus leading to a stable 50 structure. Under these conditions, the HB donor (which is a somewhat more competitive donor moiety) binds to the best acceptor site, i.e. the pyridine nitrogen, leading to a stable trimer. $\mathrm{I} / \mathrm{Br}$ then interacts with the next best acceptor (oxygen/azo nitrogen) linking the trimers into a 2-D architecture.

55 In 4,4'-azobipyridine however, the binding sites are co-linear which naturally favours the formation of infinite 1-D chains (a kinetic product) with no significant loss in energy. Under these conditions, the acceptor nitrogen atom binds to an $\mathrm{XB}$ donor as quickly as it binds to $\mathrm{HB}$ donor producing 1-D infinite chains ${ }_{60}$ constructed form alternative D-H..AA and X...A interactions.

Our results, which show a close competition between XB and $\mathrm{HB}$ donors are consistent with the work of Resnati and co-workers who designed a competitive experiment by combining 1,2-bis(4pyridyl)-ethane (BPE) as a symmetric acceptor, 1,4${ }_{65}$ diiodotetrafluorobenzene (DITFB) as halogen-bond donor and hydroquinone (HQ) as hydrogen-bond donor in a single-pot. ${ }^{26}$ Selective halogen-bonded co-crystal formation was observed, leaving the hydrogen-bond donor in a solution. The result suggests suitable XB donors can compete with certain HB donors 70 and preferentially bind to the acceptor to give stable binary cocrystals. Similarly C.M Cho et al and D.W. Bruce et al designed liquid crystals using the combination of both halogen as well as hydrogen bonds affixed on the same backbone. ${ }^{27}$

75 We have established that the orientation of the binding site in the acceptor can influence supramolecular assembly in very specific ways, and several points can be made based on the crystal structure analysis for 3,3'-and 4,4'-azobipyridine co-crystals;

1) In 3,3'-azpy co-crystals hydrogen bonding is the primary driving force $(\mathrm{HB}>\mathrm{XB})$, while in 4,4 '-azpy co-crystals both hydrogen as well as halogen bonds equally contribute in the assembly process $(\mathrm{HB} \cong \mathrm{XB})$.

2) Among the halogen atoms, iodine is a better halogenbond donor than bromine.

$853)$ There is no distinction between three types of hydrogen-bond donors (-COOH, $-\mathrm{OH}$ and $-\mathrm{CN}(\mathrm{R}) \mathrm{OH})$ in terms of binding to the azobipyridines in the presence of halogens.

\section{${ }_{90}$ Notes and references}

${ }^{a}$ Department of Chemistry, Kansas State University, Manhattan, KS, 66506,USA.E-mail: aakeroy@ksu.edu

$\dagger$ Electronic supplementary information (ESI) available: Details of the synthesis of compounds. CCDC 906983 - 906994. See 95 DOI: $10.1039 / \mathrm{b} 000000 \mathrm{x} /$ 
1 R.D.B Walsh, M.W. Bradner, S. Fleischman, L. A. Morales, B. Moulton, N. Rodriguez-Hornedo, M. J. Zaworotko, Chem. Commun. 2003, 186-187; M. Wenger and J. Bernstein, Angew. Chem., Intl. Ed. 2006, 45, 7966-7969; J-M. Lehn, Science, 2002, 295, 2400; G. R. Desiraju, Acc. Chem. Res., 2002, 35, 565-573.

2 C.B. Aakeröy, J. F. Urbina and J. Desper, Chem. Commun., 2005 , 2820-2822; T. R. Shattock, K. K. Arora, P. Vishweshwar, and M. J. Zaworotko, Cryst. Growth Des., 2008, 8, 4533-4545; C. B. Aakeröy, I. Hussain, S. Forbes and J. Desper, CrystEngComm, 2007, 9, 46-54; B. R. Bhogala, A. Nangia, Cryst. Growth Des. 2003, 3, 547-554.

3 (a) P.Vishweshwar, J. A. McMahon, M. L. Peterson, M. B. Hickey, T. R. Shattock, M. J. Zaworotko, Chem. Commun. 2005, 36, 46014603. (b) C. B. Aakeröy, J. Desper, B. A. Helfrich, CrystEngComm. 2004, 6, 19-24. (c) L. S. Reddy, A. Nangia, V. M. Lynch, Cryst. Growth Des. 2004, 4, 89-94. (d) C.B. Aakeröy, A. M. Beatty, B. A. Helfrich, M. Nieuwenhuyzen, Cryst. Growth Des. 2003, 3, 159-165.

4 K. S. Huang, D. Britton, M. C. Etter, S. R. Byrn, J. Mater. Chem. 1997, 7, 713; L. R. MacGillivray, J. L. Reid, J. A. Ripmeester, J. Am. Chem. Soc. 2000, 122, 7817; T. Friščić, L. R. MacGillivray; Chem. Commun., 2009, 773-775; I. D. H. Oswald, W. D. S. Motherwell, S. Parsons, Acta Cryst., 2004, 60, 1967-1969; P. S. Wheatley, A. J. Lough, G. Ferguson, C. Glidewell, Acta Cryst., 1999, 55, 1489-1492; A. Mukherjee, G. R. Desiraju; Chem. Commun., 2011,47, 4090-4092.

5 C. B. Aakeröy, D. J. Salmon, M. M. Smith, J. Desper, Cryst. Growth Des. 2006, 4, 1033-1042.

6 P. Metrangolo, T. Pillati, G. Resnati, A. Stevenazzi, Chem Commun, 2004, 1492-1493; P. Metrangolo, T. Pilati and G. Resnati, CrystEngComm, 2006, 8, 946-947; K. Raatikainen and K. Rissanen, CrystEngComm, 2011, 13, 6972-6977; P. Politzer, J. S. Murray, Chemistry of Halides, Pseudo-Halides and Azides, 1995, Pt.1, 1-30; T. Brinck, J. S. Murray, P. Politzer, International Journal of Quantum Chemistry, Quantum Biology Symposium, 1992, 19, 57-64.

7 P. Auffinger, F. A. Hays, E. Westhof and P. S. Ho, Proc. Natl. Acad. Sci. U. S. A., 2004, 101, 16789-16794.

8 A. R. Voth and P. S. Ho, Curr. Top. Med. Chem., 2007, 7, 13361348.

9 G. M Espallargas, Ideas in Chemistry and Molecular Sciences: Advances in Nanotechnology, Materials and Devices, 2010, 115-138.

10 P. Metrangolo, H. Neukirch, T. Pilati, G. Resnati, Acc. Chem. Res. 2005, 38, 386-395

11 C. B. Aakeröy, J. Desper, M. M. Smith, Chem. Commun., 2007, 3936; M.C. Etter, J. Phys. Chem., 1991, 95, 4601; M.C. Etter, Acc. Chem. Res. 1990, 23, 120.

12 J. W. Steed, J. L. Atwood, Supramolecular Chemistry, 2nd Ed. John Wiley \& Sons, Ltd. 2009.

13 P. Metrangolo, H. Neukirch, T. Pilati, G. Resnati, Acc. Chem. Res. 2005, 38, 386-395;

14 Halogen Bonding: Fundamentals and Applications; P. Metrangolo, G. Resnati, Eds.; Structure and Bonding; Springer: Berlin, 2008, 126

15 C. B. Aakeröy, N. Schultheiss, A. Rajbanshi, J. Desper and C. Moore. Crystal Growth\& Design, 2009, 9, 432; C. B. Aakeröy, M. Fasulo, N. Schultheiss, J. Desper, and C. Moore. J.Am Chem Soc.2007, 129, 13772,; Aakeröy, C.B. Desper, J., Helfrich, B.A., Metrangolo, P., Pilati, T., Resnati, G., Stevenazzi, A., Chem. Commun, 2007, 4236.

16 C. B. Aakeröy, A. Rajbanshi, Z. J. Li and J. Desper, CrystEngComm, 2010, 12, 4231-4239; C. B. Aakeröy, J. Desper, M. E. Fasulo, CrystEngComm, 2006, 8, 586-588.

17 C.B. Aakeröy, S. Panikkattu, B. DeHaven and J. Desper, CrystEngComm, 2013, DOI: 10.1039/C2CE26153.

18 C.B. Aakeröy, P. D. Chopade, C. Ganser and J. Desper, Chem.Commun., 2011, 47, 4688-4690.

19 APEXII v2009. 5-1, C 2009, Bruker Analytical X-ray Systems, Madison, WI.

20 COSMO v1. 60, (C) 1999 - 2009, Bruker Analytical X-ray Systems, Madison, WI.
21 SAINT v7. 60a, (C) 1997 - 2008, Bruker Analytical X-ray Systems, Madison, WI

22 SADABS v2008/1, C 2008, Bruker Analytical X-ray Systems, Madison, WI.

23 SHELXTL v2008/4, C 2008, Bruker Analytical X-ray Systems, Madison, WI.

24 A.D.Ward, V.R.Ward, E.R.T.Tiekink, Z.Kristallogr.-New Cryst.Struc, 2001, 216, 563; E.Arte, J.P.Declercq, G.Germain, M.V. Meerssche, Bull.Soc.Chim.Belg, 1980, 89, 155; B.Jerslev, S.Larsen, Acta Chem.Scand, 1992, 46, 1195. M.Tonogaki, T.Kawata, S.Ohba, Y.Iwata, I.Shibuya, Acta Crystallogr.,Sect.B:Struct.Sci, 1993, 49, 1031.

25 C.B. Aakeröy, P.D. Chopade, J. Desper, Cryst. Growth \& Des., 2011, 11, 5333-5336..

26 E. Corradi, S. V. Meille, M. T. Messina, P. Metrangolo, G. Resnati, Angew. Chem. Intl. Ed., 2000, 39, 1782-1785.

27 C. M. Cho, X. Wang, J.J. Li, C. He, J. Xu, Liquid crystals, 2012, 112; C.Präsang, H.L.Nguyen, P. N. Horton, A. C. Whitwood, Chem. Commun., 2008, 6164-6166. 